\title{
Estudio Químico del Aceite Obtenido a Partir de Siete Variedades de Soya (Glycinemax L.)
}

\author{
Jennifer J. Lafont ${ }^{(1) \star}$, Luis C. Durango ${ }^{(1)}$ y Hermes Aramendiz ${ }^{(2)}$ \\ Universidad de Córdoba, (1) Departamento de Química, laboratorio de Cinética y biocombustibles, \\ (2) Departamento de Ingeniería Agronómica, laboratorio de Fitomejoramiento, Cra 6 No 76-103, \\ Montería-Colombia.(e-mail:jenniferlafontmendoza@gmail.com; luisdurango_87@hotmail.com; \\ haramendiz@hotmail.com) \\ *autor a quien debe ser dirigida la correspondencia
}

Recibido Jun. 3, 2013; Aceptado Jul. 17, 2013; Versión final recibida Sep. 13, 2013

\begin{abstract}
Resumen
El propósito de este trabajo está encaminado al análisis químico de aceites extraídos de siete variedades de soya que fueron mejoradas genéticamente por métodos convencionales y que son denominadas Soyica $P$ 34, Cimarrona, SoySK-5, SoySk-7, S.S.K, Ariari- 1 y línea Experimental Sk. A estas variedades se les determinó el contenido de aceite, composición de ácidos grasos y algunas propiedades químicas como numero acido, valor de yodo, peróxido, saponificación y porcentaje de humedad. Estos parámetros fueron comparados entre sí para identificar las mejores variedades para la producción de biodiesel. Los resultados mostraron que el mayor porcentaje de aceite se obtuvo con la variedad Ariari-1, le siguieron la SoySk-7 y Cimarrona. En cuanto a la concentración, el ácido linoléico se encontró en mayor proporción y las propiedades químicas revelaron buena calidad de los aceites. De acuerdo a lo anterior se proponen estas variedades (Ariari-1, SoySk-7 y Cimarrona) como las más aptas para la obtención de aceites y producción de biodiesel.
\end{abstract}

Palabras clave: Glycinemax L, mejoramiento genético, variedades de soya, aceite, propiedades químicas.

\section{Chemical Study of the Oil Obtained from Seven Varieties of Soybean (Glycine max L.)}

\begin{abstract}
The purpose of this work is aimed at chemical analysis of oils extracted from seven varieties of soybeans that were genetically modified by conventional methods and that are known as Soyica P-34, Cimarrona, SoySK-5, SoySk-7, SSK, Ariari-1 and experimental Line Sk. Oil content, fatty acid composition and some chemical properties such as acid number, iodine value, peroxide, saponification and moisture content were determined from these varieties. These parameters were compared among them to identify the best varieties for the biodiesel production. The results showed that the highest percentage of oil was obtained with the Ariari-1 variety, followed by SoySk-7 and Cimarrona. In terms of concentration, linoleic acid is found in a higher proportion and chemical properties revealed the good quality of the oils. According to these findings, these varieties (Ariari-1, SoySk-7 and Cimarrona), are proposed as the most appropriate ones for obtaining oils and biodiesel production.
\end{abstract}

Keywords: Glycine max L, genetic improvement, soybean varieties, oil, chemical properties. 


\section{INTRODUCCIÓN}

La soya es una planta herbácea, que pertenece a la familia Papilonáceas (Fabáceas),cuyo ciclo vegetativo oscila de tres a siete meses, su altura varía entre 40 y $150 \mathrm{~cm}$,el tallo es rígido y erecto con tendencia a doblarse;las hojas son trifoliadas, alternas y grandes, presenta inflorescencias que agrupan flores pequeñas blancaso purpura; es una legumbre con vainas pequeñas y en cada una contiene de una a cuatro semillas esféricas oleaginosas (con un $20 \%$ de aceite) que pueden ser negras, amarillas, castañas 0 verdes(Benavidez et al., 2010; Esteves et al., 2010).La composición química del grano puede variar dependiendo de la cantidad de agua en la etapa de floración de la planta (Ghassemi y Farshbaf, 2012; Esmaeilian et al., 2012; Seth et al, 2010), la semilla de la soyaposee alto contenido nutricional comparada con otras legumbres como frijol, lentejas, garbanzos entre otros, debido a su mayor porcentaje de proteínas (38,80\% a $40,81 \%)$ y menor cantidad de carbohidratos que los anteriores, razón por el cual es utilizada en dietas balanceadas(Fresoli et al., 2012; Lokuruka, 2010). La soya también posee alto contenido de aceite, formado en su mayorproporción por ácidos grasos insaturados, los cuales son aprovechados para la producción de biodiesel, debido a que la composición química de ellos pueden influir en las propiedades de los biocombustibles(Moreira, 2013; Ramos et al., 2009; Eze, 2012).

Actualmente la soya es la oleaginosa que más se produce en el mundo, en general se ha reportado 102,993.246 ha, de las cuales el $85 \%$ de dicha área es cultivada en América (INEC, 2009) para una producción de 260,915.871 toneladas, que son utilizadas para obtener 42 millones de toneladas de aceite y proteína para consumo humano y alimentación animal (FAO, 2013); sus granos son considerados muy versátiles, ya que pueden ser consumidos como semillas de soya, brotes de soya, y asimismo pueden ser procesados para obtener derivados como: leche de soya, tofu, salsa de soya y harina (Sobral y Wagner, 2009). Además, la soya puede ser insumo de productos no comestibles, tales como cera para velas y biodiesel (INEC, 2009).

En Colombia la soya es una de las especies que además de producir aceite, es utilizada en la agroindustria para la elaboración de alimentos balanceados, obtención de aceites ymateria prima como posible fuente alternativa renovable para producción de biocombustibles (Sanz et al., 2011; Lokuruka, 2010). A través de los años se han venido desarrollando distintos procesos de mejoramiento en plantas como la soya, uno de ellos es el método convencional de cruzamiento recurrente, que se realiza con el fin de conservar y mejorar una variedad vegetal (Shuang et al., 2007).En la bibliografía se han reportado mejoramientos genéticos en la planta soya con el fin de aumentar su productividad y hacerlas más tolerantes a herbicidas (Lee y Choung, 2011), resistente a las inundaciones (Kokubun, 2012) a insectos (Sato et al., 2013), entre otros(Cargnelutti y Piffer, 2011); sin embargo es poco conocido como varia la composición química del aceite con fines biocombustibles en las semillas de soya que han sufrido este proceso. De acuerdo a lo anterior, esta investigación se encuentra orientada al estudio del aceite de las semillas de siete variedades de soya modificadas genéticamente por el método de selección de cruzamiento recurrente (Soyica P-34, Cimarrona, SoySK-5, SoySk-7, S.S.K, Ariari-1, y la línea Experimental Sk), para determinar las variedades que presenten mayor contenido de aceite, la variación en composición de ácidos grasos y evaluar algunas propiedades químicas, con el fin de seleccionar los mejores materiales para la producción de biodiesel.

\section{METODOLOGÍA}

Las semillas de soya denominadas: Soyica P-34, Cimarrona, SoySK-5, SoySk-7, S.S.K, Ariari-1 y la línea Experimental Sk, fueron modificadas genéticamente por la empresa Semillas Kamerum de la ciudad de Cartago-Colombia, utilizando el método de selección de cruzamiento recurrente para hacerlas más resistentes al herbicida glifosato; estas semillas fueron donadas a la universidad de Córdoba-Colombia; las cuales fueron sometidas a un proceso de lavado y descascarado manual, luego se maceraron y empacaron en bolsas con cierre hermético ziploc, debidamente rotuladas con el nombre de cada cultivar para ser utilizadas en la extracción del aceite y demás análisis respectivos.

El método empleado para extraer el aceite en cada variedad de soya, fue el de solventes utilizando un equipo de soxhlet con hexano como disolvente, (Sanz et al., 2011). La miscela aceite-solvente fue separada mediante roto evaporación, para recuperar el solvente y el aceite obtenido fue sometido a un proceso de desgomado para retirar las lecitinas y demás gomas presentes, este proceso consistió en calentar el aceite por separado con el agua hasta alcanzar la temperatura de $90 \mathrm{C}$, luego ambos fueron retirados del calentamiento y al aceite se le adicionó suavemente el $10 \%$ de su volumen en agua destilada caliente y se dejó en reposo por 30 minutos para esperar que las gomas precipitaran, las cuales fueron separadas posteriormente mediante centrifugación (Lafont et al., 2011), finalmente se calculó el rendimiento en aceite desgomado; y fue envasado en recipiente de vidrio ámbar a 25두 cada ensayo se realizó por triplicado. 
A los aceites obtenidos de las siete variedades de soya se les determinó el perfil de ácidos grasos(Guo et al., 2012) ylas propiedades fisicoquímicas:valor acido (Cd 3d-63), yodo (Cd 1-25), peróxido(Cd 8b-90) y saponificación (Cd 3-25); Ias cuales fueron analizadas siguiendo la metodología de laAmerican Oil Chemists' Society (AOCS, 2009); también se determinó el contenido de humedad y materia volátil para las siete variedades de soyasiguiendo el procedimiento propuesto por Ardila y León,(2010); en el cual se tomó una capsula de porcelana seca, esterilizada a $105 \mathrm{C}$ y enfriada en un desecador, se pesó y se anotó este valor, luego fueron pesados 2 gramos de cada aceite en estudio y se calentaron en una mufla a $105^{\circ} \mathrm{C}$ durante 30 min, posteriormente se enfriaron y se pesaron; este procedimiento se repitió hasta que la diferencia entre las dos pesadas no excediera el $0,05 \%$.Todas las propiedades químicas fueron realizadas inmediatamente después de la obtención del aceite y analizadas según el diseño experimental completamente al azar con tres repeticiones y prueba de media al $5 \%$ de significancia; el análisis estadístico de las variables de respuesta y la prueba de Tukey se realizó a través del programa estadístico (SAS).

Para realizar el perfil de ácidos grasos se utilizo un cromatógrafo de gases marca Agilent $6890 \mathrm{~N}$ con detector selectivo de masas Agilent 5973Ny muestreador automático de líquidos Agilent 7683, equipado con un Software - MSD Productivity Chemstation. La separación cromatográfica se llevó a cabo en una columna capilar Agilent HP-5 al 5\% de dimetilfenilsiloxano, con 0,25 mm de diámetro interno x 30m longitud nominal y $0,25 \mu \mathrm{m}$ espesor de la película; la técnica de inyección utilizada fue de flujo completo (splittles). Se empleó el método cuantitativo del patrón interno (metanol); las condiciones del método fueron rampa de calentamiento de $10 \mathrm{C} / \mathrm{min}$ hasta alcanzar $300 \mathrm{C}$, la temperatura máxima de la columna fue de $325 \mathrm{C}$ y las temperaturas del inyector y del detector alcanzaron 310C; el tiempo total de corrida fue de 60min, utilizando el helio como gas de arrastre (Guo et al., 2012).

El procedimiento empleado para la cuantificación de los ácidos grasos presentes en el aceite fue el de inyección directa, previa dilución con etanol como solvente para volatilizar las muestras y facilitar su paso a través de la columna del equipo. La identificación se realizó por comparación de los espectros de masas y los tiempos de retención de los compuestos identificados en el aceite con las bases de datos NIST, WILEY Y ADAMS, que posee el equipo, con una correspondencia del $98 \%$.

\section{RESULTADOS Y DISCUSIÓN}

En la Tabla 1 se presentan los resultados del análisis de varianza para las diferentes propiedades químicas analizadas en las siete variedades de soya, donde la media representa el valor promedio de cada propiedad en los siete cultivares; los coeficientes de variación en porcentajes (C.V (\%))reportados por el análisis de varianza, oscilaron de menor a mayor entre $0,09 \%$ para valor de saponificación y $2,18 \%$ en el valor ácido y el error experimental se encontró entre $2,7 \times 10^{-5}$ y 1,22 indicando que la diferencia existente es producto de la poca variación de la medición en la muestra y permiten sacar conclusiones confiables(Cargnelutti y Piffer, 2011).El total representa la sumatoria del tratamiento con el error; los grados de libertad (GL) fueron obtenidos de la diferencia entre el número de tratamientos menos uno $(7-1=6)$. Las medias de cuadrados del análisis de varianza para los porcentajes de aceite, los valores ácido, de yodo, peróxido, saponificación y porcentaje de humedad acusaron diferencias altamente significativas al $1 \%$ de probabilidad ${ }^{* *}$ ), estando acorde con el porcentaje de correlación $\left(R^{2}\right)$ que se encontró en el orden del $99 \%$ para todos los casos, indicando que hubo alta precisión en los datos.

Tabla 1: Análisis de Varianza de las propiedades químicas de todas las variedades de soya estudiadas

\begin{tabular}{lccccccc}
\hline $\begin{array}{l}\text { Fuente de } \\
\text { de Variación }\end{array}$ & G.L & Aceite & V. Acido & V. Yodo & V. Peróxido & V. Saponificación & Humedad \\
\hline Tratamiento & 6 & $17,658^{* *}$ & $0,032^{* *}$ & $1828,707^{* *}$ & $3,039^{* *}$ & $23,974^{* *}$ & $2,066^{* *}$ \\
Error & 14 & 0,176 & $2,7 \times 10^{-5}$ & 1,222 & 0,009 & 0,035 & 0,005 \\
Total & 20 & 17,834 & 0,032 & 1829,930 & 3,048 & 24,009 & 2,071 \\
Media & & 18,882 & 0,238 & 104,369 & 5,435 & 190,975 & 5,498 \\
C.V (\%) & & 0,594 & 2,181 & 1,059 & 1,725 & 0,099 & 1,248 \\
$R^{2}(\%)$ & 99,834 & 99,805 & 99,844 & 99,329 & 99,656 & 99,470 \\
\hline
\end{tabular}


En la Tabla 2 se describen los valores medios estimados de las propiedades químicas en los aceites de los siete cultivares de soya como: porcentaje de aceite, los valores $(V)$ ácido, yodo, peróxido y saponificación, como también el porcentaje de humedad. Las letras mayúsculas ( $A, B, C, D$ y E) representan los valores estadísticos clasificados de mayor $(A)$ a menor $(E)$ respectivamente y las letras iguales en la misma característica (porcentaje de aceite, valor acido, yodo, peróxido, saponificación y porcentaje de humedad) con diferentes cultivares, significan que los valores son estadísticamente iguales.

En cuanto al porcentaje de aceite, el mayor valor fue obtenido con la variedad Ariari-1 (22,08\%), que superó a los demás cultivares, le siguen la Cimarrona (20,41\%) y Soysk-7 $(20,49 \%)$ las cuales son estadísticamente iguales (B); es de anotar que estas tres variedades se encuentran acorde con los porcentajes de aceite reportados para la soya (20,83\%) (Esteves et al., 2010); los demás cultivares se encontraron por debajo de estos valores. En la literatura se ha demostrado que al someter algunas plantas oleaginosas como la soya, el girasol y la colza a estrés hídrico por déficit de agua en el desarrollo reproductivo de la planta (periodo de floración) disminuye el contenido de aceite y aumenta el porcentaje de proteína (Ghassemi y Farshbaf, 2012; Esmaeilian et al., 2012), lo cual posiblemente influyó en las variedades estudiadas que presentaron menor porcentaje de aceite.

Los resultados del valor ácido para todas la variedades oscilaron entre $(0,37$ y 0,11$) \mathrm{mg} \mathrm{KOH} / \mathrm{g}$, lo cual le proporciona mayor estabilidad, evitando la degradación de los mismos, estos valores fueron comparados con un estudio realizado para la soya por Eze, (2012) el cual presentó valores más elevados (2,8 mg $\mathrm{KOH} / \mathrm{g}$ ), sin embargo, en términos generales un aceite que presente valores por debajo del $5 \mathrm{mg} \mathrm{KOH} / \mathrm{g}$ de ácidos grasos libres puede facilitar el proceso de producción de biocombustibles y reducir la formación de jabones, mejorando su calidad,(Burín et al., 2010), los aceites de las siete variedades de soya estudiadas cumplen con este parámetro de calidad.

Los valores de yodo obtenidos en las siete variedades de soya, oscilaron entre $(139,50$ y 105,12)cg I / geste parámetro mide la cantidad de yodo absorbido por gramo de grasa o aceite y está relacionado con numero de insaturaciones presentes en los ácidos grasos, de tal forma que a mayor número de dobles enlaces este valor se incrementa y facilita el proceso de oxidación debido a la formación de epóxidos responsables de la rancidez en los aceites. Los valores de yodo obtenidos en este trabajo son comparables con otros estudios de aceites de soya $(123,42 \mathrm{cg} \mathrm{I} \mathrm{/} \mathrm{g),} \mathrm{coco}(129,48 \mathrm{cg} \mathrm{I} \mathrm{/} \mathrm{g)} \mathrm{y} \mathrm{melón}(126,90 \mathrm{cg} \mathrm{I} / \mathrm{g})$ reportados por Eze, (2012); estos resultados están acorde con los valores de este parámetro para la producción de biodiesel que para la soya oscila entre $(121-143) \mathrm{cg} \mathrm{I} / \mathrm{g}$, similares a las del girasol que se encuentran en el rango de (127-142)cg I/g (Avellaneda, 2010).

Tabla 2: Valores medios estimados de las propiedades químicas de los aceites en los cultivares de soya

\begin{tabular}{|c|c|c|c|c|c|c|c|c|c|}
\hline Características & Soysk-5 & Soysk-7 & S.S.K & $\begin{array}{l}\text { Cultivares } \\
\text { Cimarrona }\end{array}$ & Línea ex & p-Sk & P-34 & & Ariari-1 \\
\hline Aceite (\%) & $17,87 \mathrm{D}$ & $20,49 \quad B$ & $18,11 \quad D$ & $20,41 \quad B$ & 14,58 & E & 18,62 & C & $22,08 \quad A$ \\
\hline $\begin{array}{l}\text { V. Acido } \quad \text { (mg } \\
\mathrm{KOH} / \mathrm{g})\end{array}$ & $0,25 \mathrm{C}$ & $0,35 \quad B$ & $0,11 \quad E$ & $0,11 \quad E$ & 0,23 & $\mathrm{D}$ & 0,37 & $A$ & $0,23 \quad D$ \\
\hline $\begin{array}{l}\text { V. Yodo } \\
\text { (cg I/g) }\end{array}$ & $120,12 B$ & $108,68 \mathrm{C}$ & $139,50 \mathrm{~A}$ & $119,34 \mathrm{~B}$ & 107,30 & $\mathrm{C}$ & 105,12 & & $108,02 \mathrm{C}$ \\
\hline $\begin{array}{l}\text { V. Peróxido } \\
\left(\text { meq } \mathrm{O}_{2} / \mathrm{kg}\right)\end{array}$ & $7,51 \mathrm{~A}$ & $5,24 \quad C$ & $5,57 \quad B$ & 4,52 & 4,89 & $\mathrm{D}$ & 4,85 & $E$ & $5,60 \quad B$ \\
\hline $\begin{array}{l}\text { V. } \\
\text { Saponificación(mg } \\
\mathrm{KOH} / \mathrm{g})\end{array}$ & $192,89 \mathrm{~B}$ & $192,39 \mathrm{~B}$ & $190,81 \mathrm{C}$ & $185,80 \mathrm{E}$ & 190,45 & C & 194,69 & $A$ & $189,79 \mathrm{D}$ \\
\hline Humedad (\%) & $5,52 \quad C$ & $6,41 \quad B$ & $4,25 \quad E$ & $4,27 \quad E$ & 5,16 & D & 6,72 & A & 5,14 \\
\hline
\end{tabular}

El valor de peróxido mide el grado de oxidación primaria que ha sufrido el aceite, experimentalmente se encontró que en las siete variedades, este valor oscilo entre $(7,51-4,52)$ meq $\mathrm{O}_{2} / \mathrm{kglos}$ cuales son considerados bajos,con respecto a otros estudios de aceite de soya reportados por Eze, (2012) con valor de 16,32 meq $\mathrm{O}_{2} / \mathrm{kg}$ y similares a los aceites de palma $\left(7,96\right.$ meq $\left.\mathrm{O}_{2} / \mathrm{kg}\right)$ y maní $\left(6,20\right.$ meq $\left.\mathrm{O}_{2} / \mathrm{kg}\right)$; en 
general se puede decir que los aceites con valor de peróxido bajo son poco susceptibles a su deteriorooxidativo.El valor de saponificación se define como el número de mg de $\mathrm{KOH}$ necesarios para neutralizar los ácidos grasos provenientes de la hidrólisis de un gramo de aceite; estos valores oscilaron entre $(194,69$ - 185,80) mg KOH / g, los cuales son similares a los encontrados por Eze, (2012) el cual reporta 195,63 $\mathrm{mg} \mathrm{KOH} \mathrm{/} \mathrm{g,} \mathrm{siendo} \mathrm{más} \mathrm{cercano} \mathrm{el} \mathrm{aceite} \mathrm{de} \mathrm{la} \mathrm{variedad} \mathrm{P-34;} \mathrm{datos} \mathrm{similares} \mathrm{fueron}$ obtenidos para el aceite de la semilla del melón con 187,0 mg KOH / g; y de la papaya con 198,50 mg KOH / g (Kalou et al., 2011),lo cual indica que se requiere de mayor cantidad de álcali para neutralizar los ácidos grasos libres presentes, siendo un factor importante para la calidad de los aceites.

El análisis del porcentaje de humedad mide el contenido de agua presente en los aceites y aunque estos son inmiscibles, puede existir en forma de emulsión; la humedad favorece la hidrólisis, especialmente en los aceites con valor ácido elevado; en este trabajo se encontró que los valores de humedad oscilaron entre $(6,72-4,25) \%$, los cuales fueron menores a los obtenidos en otro trabajo con aceite crudo de soya (Seth et al., 2010) donde reportan valores de $(13,4$ y 12,6)\%;es de anotar que los resultados de porcentaje de humedad en las siete variedades, estuvieron acorde con los datos de valor ácido, de tal forma que en ambas características el mayor valor se obtuvo con la variedad P-34 y el menor con S.S.K, en todas las variedades los valores fueron bajos disminuyendo el riesgo de hidrolisis lo cual favorece la calidad de los aceites.

En la Tabla 3 se presentan los valores promedio y desviaciones estándar de los porcentajes de ácidos grasos $(\dot{x} \pm \sigma) \%$ presentes en los siete cultivares de soya, encontrándose poca variación entre ellos y hubo prevalencia dellinoléico (C 18:2) ácido graso diinsaturado con valores entre $(52,55$ - 53,01)\%, le sigue el oleico (C 18:1), monoinsaturado con (22,06-23,51)\%, luego el palmítico (C 16:0) saturado con (11,36 $12,04) \%$,el linolénico (C 18:3) poliinsaturado entre $(5,45-6,08) \%$ y en menor proporción el esteárico (C 18:0) ácido graso saturado entre $(4,02-5,25) \%$; en general se puede decir que el porcentaje de ácidos grasos insaturados fue mayoritario, se encontraron en el rango de $(80,17-82,91) \%$, estando los saturados en menor proporción $(15,38-17,25) \%$, lo cual indica que estos aceites son líquidos a temperatura ambiente y presentan bajo punto de fusión (Moreira, 2013). Los porcentajes de ácidos grasos encontrados estuvieron acordes con otros estudios reportados para el aceite de soya, cuyos valores fueron: linoléico (53,46\%), oléico $(23,47 \%)$, palmítico $(11,44 \%)$, linolénico $(6,64 \%)$ y el esteárico $(4,14 \%)$; resultados similares se encontraron con otros aceites como el de maíz, algodón y girasol (Giakoumis, 2013).

Tabla 3: Porcentajes de ácidos grasos obtenidos en las siete variedades de soya estudiadas

\begin{tabular}{|c|c|c|c|c|c|}
\hline \multirow[b]{2}{*}{ Cultivares } & \multicolumn{5}{|c|}{ Ácidos grasos $(\dot{\mathrm{x}} \pm \sigma) \%$} \\
\hline & Palmítico & Esteárico & Oléico & Linoléico & Linolénico \\
\hline Soysk-5 & $11,29 \pm 0,2$ & $4,11 \pm 0,1$ & $23,35 \pm 0,5$ & $53,01 \pm 0,3$ & $5,91 \pm 0,4$ \\
\hline Soysk-7 & $11,98 \pm 0,7$ & $4,88 \pm 0,3$ & $22,93 \pm 0,4$ & $52,55 \pm 0,2$ & $5,67 \pm 0,6$ \\
\hline S.S.K & $11,36 \pm 0,6$ & $4,02 \pm 0,5$ & $23,51 \pm 0,3$ & $53,32 \pm 0,1$ & $6,08 \pm 0,5$ \\
\hline Cimarrona & $11,43 \pm 0,3$ & $4,25 \pm 0,7$ & $23,22 \pm 0,6$ & $52,98 \pm 0,8$ & $5,86 \pm 0,1$ \\
\hline Línea exp-SK & $12,04 \pm 0,9$ & $4,92 \pm 0,2$ & $22,71 \pm 0,1$ & $52,64 \pm 0,5$ & $5,78 \pm 0,3$ \\
\hline P-34 & $12,00 \pm 0,1$ & $5,25 \pm 0,5$ & $22,06 \pm 0,2$ & $52,66 \pm 0,4$ & $5,45 \pm 0,2$ \\
\hline Ariari-1 & $11,92 \pm 0,5$ & $4,76 \pm 0,6$ & $22,94 \pm 0,1$ & $52,63 \pm 0,2$ & $5,52 \pm 0,1$ \\
\hline
\end{tabular}

La composición de los ácidos grasos en los aceites no es alterada en el proceso de transesterificación para convertirlos a biodiesel, por lo tanto los esteres formados presentan el mismo perfil del aceite vegetal usado en el proceso (Ramos et al., 2009), es por ello que la longitud de la cadena y el grado de insaturaciones de los ácidos grasos influyen en la composición química del biodiesel y determinan sus propiedades; los principales ácidos grasos de los aceites son los que poseen entre 16 y 18 carbonos, como palmítico, esteárico, oleico, linoléico y linolénico; en cuanto al grado de insaturaciones, los aceites cuya composición química poseen ácidos grasos insaturados en mayor proporción al ser convertidos en biodiesel se espera que presente baja resistencia a la oxidación, por lo que se debe evaluar la adición de antioxidantes y su almacenamiento, sin embargo a baja temperatura se facilita el flujo adecuado de combustible, ya que disminuyen la obstrucción del filtro frio, punto de nube y de fluidez mejorando estas propiedades de baja temperatura (Moreira, 2013; Ramos et al., 2009). 
Por el contrario aquellos aceites que tienen ácidos grasos saturados en mayor proporción se espera que presenten buena estabilidad oxidativa y que aumenten las dificultades con el flujo adecuado del combustible; este comportamiento contradictorio entre las propiedades de calidad del biodiesel (estabilidad oxidativa y propiedades de baja temperatura) genera dificultades para encontrar el aceite que satisfaga ambas propiedades para la producción de biodiesel, se ha encontrado que los aceites que presentan alta concentración de ácido oléico (C18:1) favorecen ambas propiedades (Ramos et al., 2009), sin embargo aquellos aceites cuya composición presente exceso de otros ácidos grasos insaturados como el linoléico obtenido en este trabajo para las siete variedades de soya, pueden ser usados en mezclas con otros biodiesel que contengan altos ácidos grasos saturados como el de palma, para mejorar la estabilidad oxidativa (Moreira, 2013).

\section{CONCLUSIONES}

Después de analizar los resultados obtenidos en los siete cultivares de soya, se puede concluir lo siguiente: 1). La variedad Ariari -1 presentó mayor porcentaje de aceite con relación a las demás variedades, de igual forma la Soysk-7 y la Cimarrona exhibieron valores altos acorde con los reportados en la literatura para la soya. 2). El contenido de ácidos grasos en todas las variedades presentaron poca variación entre ellos, encontrándose alto porcentaje de insaturados especialmente el ácido linoléico. 3). Las propiedades químicas medidas en este trabajo estuvieron acordes con otros estudios realizados en aceites de soya, reflejandobuena calidad delos aceites y bajo grado de rancidez o deterioro del mismo. 4) De acuerdo a lo anterior se puede proponer las variedades Ariari -1, Soysk-7 y Cimarrona como las mejores para la producción de biodiesel.

\section{AGRADECIMIENTOS}

Los autores agradecen a la Empresa Semillas Kamerum -Colombia por facilitar las semillas en estudio.

\section{REFERENCIAS}

(AOCS), American Oil Chemists' Society Official Methods and Recommended Practices of the AOCS. 6 ed. Press, Washington (2009).

Ardila, J. y A. León, Caracterización del biodiesel producido a partir de aceite de higuerilla y evaluación de las variables de proceso sobre el porcentaje de glicerina, Virtualpro: 110, 1-20 (2010).

Avellaneda, F, Producción y caracterización de biodiesel de palma y de aceite reciclado mediante un proceso batch y un proceso contínuo con un reactor helicoidal, Tesis Doctoral, Departamento de Ingeniería química, Universitat de Rovira I Virgili, Tarragona, España (2010).

Benavidez, A., R. Hernández., R. Homero., A. Sandoval, Tratado de Botánica Económica Moderna, Universidad Autónoma Agraria Antonio Narro, Buenavista, Saltillo, Coah-México, (2010).

Burín, M., G. Rodríguez., M. Ribeiro, Plantas de biodiesel de aceite vegetal usado, condiciones técnicas para la aprobación INTI de plantas discontinuas para autoconsumo; Ministerio de Industria, Secretaría de Industria y Comercio, Buenos Aires, Argentina(2010).

Cargnelutti, A., Piffer, E., Estimativa do número de repetições para a avaliação de caracteres de produtividade e de morfologiaemgenótipos de soja, ComunicataScientiae: 2(1), 25-33 (2011).

Esmaeilian, Y., Sirousmehr, A., Asgripour, M., Amiri, E., Comparison of sole and combined nutrient application on yield and biochemical composition of sunflower under water stress, International Journal of applied science and technology: 2 (3) 214-220 (2012).

Esteves, E., H. Duarte., F. Esteves., J. Bressan., M. Brunoro., Chemical composition of a soybean cultivar lacking lipoxigenases (LOX2 and LOX3), Food Chemistry: 122, 238-242 (2010).

Eze, S., Physico-chemical properties of oil from some selected underutilized oil seeds available for biodiesel preparation, African Journal of Biotechnology:11 (42),10003-10007 (2012).

FAO, Organización de las Naciones Unidas para la Alimentación y la Agricultura, International Conference on open data for agriculture, Washington, D.C - United Stated of America2013. http://faostat.fao.org (con acceso 18-04-2013). 
Fresoli, D., P. Beret., Y. Hirschfeld, PROT 12 FCA: Nuevo cultivar de soja no transgénico con alta proteína, Ciencia, Docencia y Tecnología: 23 (45), 187-194 (2012).

Ghassemi-Golezani, K. y S. Farshbaf-Jafari, Influence of water deficit on oil and protein accumulation in soybean grains, International Journal of Plant, Animal and Environmental Sciences: 2(3), 46-52 (2012).

Giakoumis, E.G., A statistical investigation of biodiesel physical and chemical properties, and their correlation with the degree of unsaturation, Renewable Energy: 50, 858-878 (2013).

Guo, L.X., y otrossieteautores, Fatty acid composition of lipids in wild cordycepssinensis from habitats in China, Biomedicine \& Preventive Nutrition: 2, $42-50$ (2012).

INEC, Instituto Nacional de Estadística y Censo. Sistema agroalimentario de la soya, Ecuador, 1-24, (2009). http://www.ecuadorencifras.com/sistagroalim/pdf/Soya.pdf.

Kalou, G., y otros ocho autores, Physico-chemical properties of seed oil from papaya (cariaca papaya) and the kinetics of degradation of the oil during heating, Advance Journal of food Science and technology: 3 (1), 45-49 (2011).

Kokubun, M., Geneticand cultural improvement of soybean for waterlogged conditions in Asia, Field Crops Research: 1-5 (2012).

Lafont, J.J., M.S. Pàez.,A.A. Portacio, Extracción y Caracterización Fisicoquímica del Aceite de la Semilla (Almendra) del Marañón (Anacardiumoccidentale L), Información Tecnológica: 22(1), 51-58 (2011).

Lee, J., M, Choung.,Nondestructive determination of herbicide-resistant genetically modifiedsoybean seeds using near-infrared reflectance spectroscopy, Food Chemistry 126, 368-373(2011).

Lokuruka, M., Soybean nutritional properties: The good and the bad about soy foods consumption-a review, African Journal of food agriculture nutrition and development: 10 (4) 2439-2459 (2010).

Moreira, E., Principales características de las materias primas utilizadas en la producción de biodiesel: la influencia del contenido y la concentración de los ácidos grasos, Ingenium, Facultad de Ingeniería: 25, 53-61 (2013).

Ramos, M., Fernández, C., Casas, A., Rodríguez, L., Pérez, A., Influence of fatty acid composition of raw materials on biodiesel properties, Bioresource Technology: 100 (1), 261-268 (2009).

Sanz, J.F. y otros siete autores, Life Cycle Assessment (LCA) of the biofuel production process from sunflower oil, rapeseed oil and soybean oil, Fuel Processing Technology: 92 190-199 (2011).

Sato, D., H, Akashia., M, Sugimoto., M, Tomita., T, Soga., Metabolomic profiling of the response of susceptible and resistant soybean strains to foxglove aphid, Aulacorthumsolani Kaltenbach, Journal of Chromatography B, 925, 95-103 (2013).

Seth, S., Agrawal, Y., Ghosh, P., Jayas, D., Effect of Moisture Content on the Quality of Soybean Oil and Meal Extracted by Isopropyl Alcohol and Hexane, Food and Bioprocess Technology: 3 (1), 121-127 (2010).

Shuang, Z., Z, Meng., J, Chun., Y, Chun., L, Bing, Study on quality improvement effect and separate character of soybean male sterile (MS1) recurrent selection population, Agricultural sciences in China: 6 (5) 545-551 (2007).

Sobral, P., y J. Wagner, Relación entre la Composición y la Actividad Antitríptica de Sueros de Soja y Tofu y Comportamiento Térmico de sus Proteínas Aisladas, Información Tecnológica: 20 (5), 65-73 (2009). 
